\title{
Study of personal qualities and EEG activity in a stop signal paradigm in residents of northern regions
}

Tatiana Asakhova

NSU, Novosibirsk, Russia

t.astakhova@g.nsu.ru

Alexandra Karpova

North-Eastern Federal University, Yakutsk, Russia

karpova74@list.ru

Alexander Savostyanov

State Scientific-Research Institute of

Physiology \& Basic Medicine,

Novosibirsk, Russia

ICG SB RAS, Novosibirsk, Russia

NSU, Novosibirsk, Russia

alexander.savostyanov@gmail.com

\author{
Alexander Saprigyn \\ State Scientific-Research Institute of \\ Physiology \& Basic Medicine, \\ Novosibirsk, Russia \\ saprigyn@mail.ru \\ Natalya Borisova \\ North-Eastern Federal University, \\ Yakutsk, Russia \\ borinat@yandex.ru
}

Sergey Tamozhnikov

State Scientific-Research Institute of

Physiology \& Basic Medicine,

Novosibirsk, Russia

stam@physiol.ru

Elena Afanaseva

North-Eastern Federal University,

Yakutsk, Russia

eb.afanaseva@mail.ru

\begin{abstract}
It is known that the adaptation to new conditions depends on personality traits. The results obtained showed that the lower level of anxiety and neurotism, the better behavioural control and adaptation. Also, the adaptation level is the lowest right after the move to another place, and it grows by the time along with the growth of behavioral control.
\end{abstract}

Keywords - adaptation, personality traits, stop signal paradigm, SSP, EEG.

\section{Aim}

The aim of the study was to identify correlates of personality traits in the context of SSP in local people and migrants who live in polar or subpolar regions of the Republic of Sakha (Yakutia).

\section{Methods}

Four groups of young healthy people participated in the study: 1) people who permanently live in Novosibirsk (150 people, 70 men, average age $23 \pm 4$ y.o.); 2) people who permanently live in Yakutsk ( 85 people, 39 men, $24 \pm 3$ y.o.); $3)$ people who permanently live in the Arctic regions of Yakutia (50 people, 21 men, $26 \pm 4$ y.o.); 4) labor migrants who moved to Yakutia from the southern countries (50 people, all men, $23 \pm 2$ y.o.). The examination of group of migrants was carried out twice - immediately after the move and six months after. Stop signal paradigm methodology was chosen in order to observe the brain activity in conditions of behavior control. In SSP experiment participants had to quickly press one of the two buttons after the target signal onset (130 trials), or suppress the movement that had already begun (33 trials). EEG was recorded by 64 channels amplifier - Brain Products, Germany. Event related potentials (ERP) were used as a functional changes indicator of tasks performance. Markers of the "Big Five" (D. Goldberg's questionnaire) were considered as personality characteristics indicators.

\section{Results}

The questionnaires results analysis showed differences between four groups of participants in terms of neurotism, personal anxiety, extraversion and compliance. Behavioral indicators of motor control were the best in local people of Yakutia (both groups), average for Novosibirsk and the worst in migrants at the first examination. The quality of motor control increased in the second examination. The quality of motor control positively correlated with indices of neuroticism and anxiety among the indigenous inhabitants of Yakutia (both groups), negatively correlated among migrants and didnot correlate with neuroticism among residents of Novosibirsk. EEG in the group of local people of Yakutia positively correlated between the P300 amplitude, the potential of readiness and the level of neurotism and anxiety, while migrants showed negative correlations of these indicators, while reliable correlation was not found in the participants from Novosibirsk.

\section{Conclusion}

Acute stress during adaptation to the north climate among migrants caused a decrease in the ability to control their behavior. It also resulted an increase in the neuroticism and anxiety. In contrast, in local Yakuts the increased neurotism and anxiety were associated with the increase in the ability to control behavior.

\section{ACKNOWLEDGMENT}

The study was supported by RFFI grants No. 18-29-13027 and 18-415-140021. 